\title{
OPERADIC TENSOR PRODUCTS AND SMASH PRODUCTS
}

\author{
J. P. MAY
}

\begin{abstract}
Let $k$ be a commutative ring. $E_{\infty} k$-algebras are associative and commutative $k$-algebras up to homotopy, as codified in the action of an $E_{\infty}$ operad; $A_{\infty} k$-algebras are obtained by ignoring permutations. Using a particularly well-behaved $E_{\infty}$ algebra, we explain an associative and commutative operadic tensor product $\otimes$ that effectively hides the operad: an $A_{\infty}$ algebra or $E_{\infty}$ algebra $A$ is defined in terms of maps $k \longrightarrow A$ and $A \otimes A \longrightarrow A$ such that the obvious diagrams commute, and similarly for modules over $A$. This makes it little more difficult to study these algebraic objects than it is to study their classical counterparts. We also explain a topological analogue of the theory. This gives a symmetric monoidal category of modules over the sphere spectrum $S$ whose derived category is equivalent to the classical stable homotopy category. The existence of this category allows the wholesale importation of algebraic techniques into stable homotopy theory.

There will not be time to go into this, but the algebraic theory has applications to mixed Tate motives in algebraic geometry and the topological theory has applications to the construction and study of $M U$-module spectra, the construction of generalized Künneth and universal coefficient spectral sequences, a construction of the algebraic $K$-theory of $S$-algebras that includes Quillen's algebraic $K$-theory of discrete rings and Waldhausen's algebraic $K$-theory of spaces, a construction of the topological Hochschild homology of an $S$-algebra that generalizes Bökstedt's THH, and a completion theorem for equivariant complex cobordism and any of its modules analogous to the Atiyah-Segal completion theorem in equivariant $K$-theory.
\end{abstract}

\section{The Category of $\mathbb{C}$-modules and the product $\bigotimes$}

Let $\mathscr{C}$ be an operad in a cocomplete symmetric monoidal category $\mathscr{S}$ with product $\otimes$ and unit $\kappa$. We are thinking of the category of differential graded modules over a commutative ring $k$ and will restrict to it shortly. In general, $\mathbb{C}=\mathscr{C}(1)$ is a monoid in $\mathscr{S}$. In our algebraic context, this means that $\mathbb{C}$ is a DGA. We call left $\mathbb{C}$-objects $\mathbb{C}$-modules in any case. In the algebraic situation, if $\mathscr{C}$ is unital and the augmentation $\epsilon: \mathbb{C} \rightarrow k$ is a quasi-isomorphism, then the derived categories $\mathscr{D}_{k}$ and $\mathscr{D}_{\mathbb{C}}$ are equivalent.

Via instances of the structural maps $\gamma$, we have a left action of $\mathbb{C}$ and a right action of $\mathbb{C} \otimes \mathbb{C}$ on $\mathscr{C}(2)$, and these actions commute with each other. Thus we have a bimodule structure on $\mathscr{C}(2)$. Let $M$ and $N$ be left $\mathbb{C}$-modules. Clearly $M \otimes N$ is a left $\mathbb{C} \otimes \mathbb{C}$-module via the given actions. This makes sense of the following definition of the "operadic tensor product $\otimes "$.

1991 Mathematics Subject Classification. Primary 18-02, 55-02; Secondary 18C99, 55P42.

This talk is largely based on Part V of [7] and on [5], to which the interested reader is referred for details and more complete references.

The author was supported in part by NSF Grant \#DMS-9423300. 
Definition 1.1. For $\mathbb{C}$-modules $M$ and $N$, define $M \otimes N$ to be the $\mathbb{C}$-module

$$
M \otimes N=\mathscr{C}(2) \otimes_{\mathbb{C} \otimes \mathbb{C}} M \otimes N .
$$

This definition deserves more study than it has been given. In special cases, it has led to interesting algebraic results, which are joint work with Igor Kriz and which I shall describe. These are largely motivated by applications to mixed Tate motives, but I will not go into that. An analogous operadic smash product has led to really rather spectacular results in topology, which are joint work with Elmendorf, Kriz, and Mandell [5]. I will give an introduction to the parallel topological theory at the end.

We concentrate on algebra. Here we have an operadic Hom functor Hom ${ }^{\circledR}$ on $\mathbb{C}$-modules to go with the operadic tensor product. Since its precise definition is dictated by the adjunction, I won't bother writing it down.

Lemma 1.2. There is a natural adjunction isomorphism

$$
\mathscr{M}_{\mathbb{C}}(M \otimes N, P) \cong \mathscr{M}_{\mathbb{C}}\left(M, \operatorname{Hom}^{\unrhd}(N, P)\right) .
$$

The product $\otimes$ is always commutative.

Lemma 1.3. There is a canonical commutativity isomorphism of $\mathbb{C}$-modules

$$
\tau: M \otimes N \longrightarrow N \otimes M \text {. }
$$

Proof. Use the action of the transposition $\sigma \in \Sigma_{2}$ on $\mathscr{C}(2)$ together with the transposition isomorphisms $\mathbb{C} \otimes \mathbb{C} \rightarrow \mathbb{C} \otimes \mathbb{C}$ and $M \otimes N \rightarrow N \otimes M$.

Associativity is more subtle and requires an exceptionally well-behaved operad. Although we are working algebraically, the following basic result comes from topology.

Theorem 1.4. There is an $E_{\infty}$ operad $\mathscr{C}=C_{*}(\mathscr{L})$, where $\mathscr{L}$ is the "linear isometries operad", for which there is a canonical associativity isomorphism of $\mathbb{C}$-modules

$$
(L \otimes M) \otimes N \cong L \otimes(M \otimes N) .
$$

In fact, for any $j$-tuple $M_{1}, \ldots, M_{j}$ of $\mathbb{C}$-modules, there is a canonical isomorphism

$$
M_{1} \otimes \cdots \otimes M_{j} \cong \mathscr{C}(j) \otimes_{\mathbb{C}^{j}}\left(M_{1} \otimes \cdots M_{j}\right)
$$

where the iterated product on the left is associated in any fashion. For $j \geq 2$, the $j$-fold $\otimes$-power $\mathbb{C}^{\bigotimes j}$ is isomorphic to $\mathscr{C}(j)$ as a $\left(\mathbb{C}, \mathbb{C}^{j}\right)$-bimodule.

It is also true that $\mathscr{C}(j)$ is isomorphic to $\mathbb{C}$ as a left $\mathbb{C}$-module. These properties are quite miraculous. I will explain why they are true later, after describing some of the implications. We restrict attention to this particular operad $\mathscr{C}$ from now on. As long as we are considering $E_{\infty}$ operads, there is no loss of generality: algebras and their modules over other $E_{\infty}$ operads can be converted functorially to algebras and their modules over $\mathscr{C}$.

Note that $k$ is a $\mathbb{C}$-module via the augmentation $\mathbb{C} \rightarrow k$. The degeneracy map $\sigma_{1}: \mathscr{C}(2) \rightarrow \mathscr{C}(1)=\mathbb{C}$ induces a natural unit map.

Lemma 1.5. There is a natural map of $\mathbb{C}$-modules $\lambda: k \otimes N \rightarrow N$.

This map is not an isomorphism, but another special property of the linear isometries operad implies that it is usually a quasi-isomorphism.

Lemma 1.6. $\sigma_{1}: \mathscr{C}(2) \rightarrow \mathbb{C}$ is a homotopy equivalence of right $\mathbb{C}$-modules. 
There is not time to describe it today, but there is a theory of cell modules over DGA's that is just like the topological theory of CW complexes or CW spectra, except much simpler. Briefly, free modules on suspensions of $k$ serve as analogs of spheres and thus as the domains of attaching maps for algebraic cones. We pass to derived categories by approximating general modules by quasi-isomorphic cell modules. Via such cell approximations, the functors $\otimes$ and $\operatorname{Hom}^{\otimes}$ induce a derived tensor product $\stackrel{L}{\otimes}$ and a derived Hom functor $R \mathrm{Hom}^{\otimes}$ on $\mathscr{D}_{\mathbb{C}}$. These functors are nicely related to the derived tensor product and Hom functors on $k$-modules.

Proposition 1.7. If $N$ is a cell module, then $\lambda: k \otimes N \rightarrow N$ is a quasi-isomorphism, the functor $M \otimes N$ of $M$ preserves exact sequences and quasi-isomorphisms, and the $k$-module $M \otimes N$ is naturally quasi-isomorphic to $M \otimes N$. Therefore the equivalence of derived categories $\mathscr{D}_{\mathbb{C}} \rightarrow \mathscr{D}_{k}$ induced by the forgetful functor from $\mathbb{C}$-modules to $k$-modules carries $\stackrel{L}{\otimes}$ to $\stackrel{L}{\otimes}$.

Proposition 1.8. If $N$ is any $\mathbb{C}$-module, then the adjoint $N \longrightarrow \operatorname{Hom}^{\bigotimes}(k, N)$ of $\lambda$ is a quasi-isomorphism and the functor $\operatorname{Hom}^{\bigotimes}(M, N)$ of $M$ preserves exact sequences of cell $\mathbb{C}$-modules. If $M$ is a cell $\mathbb{C}$-module, then the functor $\operatorname{Hom}^{\circledR}(M, N)$ of $N$ preserves exact sequences and quasi-isomorphisms, and the $k$-module $\operatorname{Hom}^{\bigotimes}(M, N)$ is quasi-isomorphic to $\operatorname{Hom}(M, N)$. Therefore the equivalence of derived categories $\mathscr{D}_{\mathbb{C}} \rightarrow \mathscr{D}_{k}$ induced by the forgetful functor carries $R \mathrm{Hom}^{\bigotimes}$ to $R$ Hom.

Remark 1.9. It would be of interest to construct an $E_{\infty}$ operad with the properties that we have quoted by purely algebraic methods. One further property is out of reach. We would like $\lambda: k \otimes k \rightarrow k$ to be an isomorphism. The indecomposable quotient $\mathscr{C}(2) \otimes_{\mathscr{C}(1) \otimes \mathscr{C}(1)} k$ would then be $k$. We have required that $\mathscr{C}(j)_{n}=0$ for $n<0$, and it is natural to require further that $\mathscr{C}(1)_{0}=k$. This property would then imply that $\mathscr{C}(2)_{0}=k$, contradicting the requirement that $\mathscr{C}(2)$ be $\Sigma_{2}$-free.

\section{A NeW DEscription of $A_{\infty}$ AND $E_{\infty}$ Algebras And MOdules}

We consider $\mathscr{C}$-algebras and their modules, where $\mathscr{C}=C_{*}(\mathscr{L})$. We call these $A_{\infty}$ algebras when we ignore permutations and $E_{\infty}$ algebras when we retain them.

Restricting the action to $j=1$, we see that an $A_{\infty}$ algebra is a $\mathbb{C}$-module with additional structure. From $j=0$ we obtain a unit $\eta: k \longrightarrow A$ and from $j=2$ we obtain a product $\phi: A \otimes A \rightarrow A$. The rest of the operad action is actually determined by this portion of it. The following result is the analog of a theorem first discovered in a much deeper topological context.

Theorem 2.1. An $A_{\infty}$ algebra $A$ determines and is determined by a $\mathbb{C}$-module with a unit map $\eta: k \longrightarrow A$ and a product map $\phi: A \otimes A \rightarrow A$ such that the following diagrams commute:
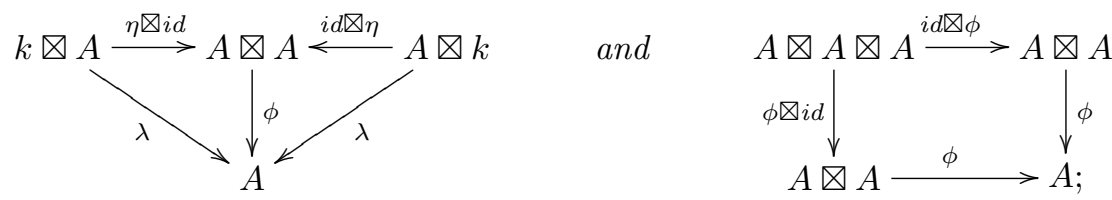
$A$ is an $E_{\infty}$ algebra if the following diagram also commutes:

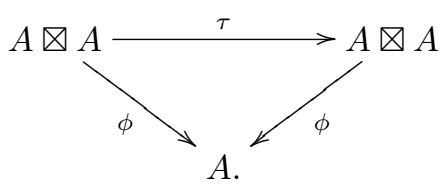

Although I will not have time to go into detail, there is an even simpler and more convenient way to package these reinterpretations of $A_{\infty}$ and $E_{\infty}$ algebras.

Proposition 2.2. The category of $\mathbb{C}$-modules under $k$ admits a variant $\square$ of the product $\square$ with respect to which it is symmetric monoidal with unit $k$. An $A_{\infty}$ or $E_{\infty}$ algebra is precisely a monoid or commutative monoid in this category.

To illustrate the force of this result, I will give a formal consequence. Recall that the tensor product of commutative DGA's is their coproduct in the category of commutative DGA's. The proof consists of categorical diagram chases that now carry over to our more general context.

Corollary 2.3. Let $A$ and $B$ be $A_{\infty}$ algebras. Then $A \square B$ is an $A_{\infty}$ algebra. If $M$ is an $A$-module and $N$ is a $B$-module, then $M \otimes N$ is an $A \square B$-module. If $A$ and $B$ are $E_{\infty}$ algebras, then $A \boxminus B$ is an $E_{\infty}$ algebra and is the coproduct of $A$ and $B$ in the category of $E_{\infty}$ algebras.

We have a similar reinterpretation of the notion of an $A$-module.

Theorem 2.4. Let $A$ be an $A_{\infty}$ or $E_{\infty}$ algebra. An $A$-module is a $\mathbb{C}$-module $M$ together with a map $\mu: A \otimes M \rightarrow M$ such that the following diagrams commute:
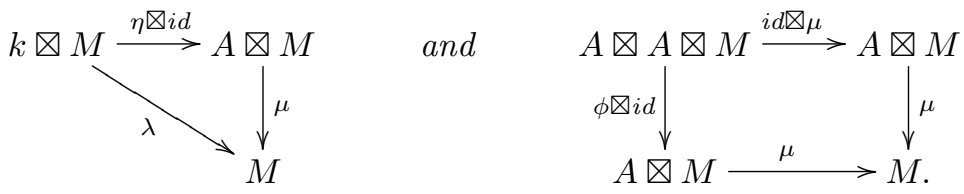

When $A$ is an $E_{\infty}$ algebra, this implies that we obtain the same $A$-modules for $A$ regarded as an $E_{\infty}$ algebra as for $A$ regarded by neglect of structure as an $A_{\infty}$ algebra. This is far from obvious with the original operadic definitions.

\section{3. $U(A)$ AND THE DERIVED CATEgory of $A$-Modules}

Fix an $A_{\infty}$ algebra $A$. The category of $A$-modules is isomorphic to the category of $U(A)$-modules. It therefore enjoys all of the formal properties that are familiar from the context of modules over a non-commutative DGA. Here we are thinking of $A$-modules as defined in the ground category of $k$-modules. However, the ideas above dictate that we sometimes change our point of view and regard $A$-modules as defined in the category of $\mathbb{C}$-modules. Since $U(k)=\mathbb{C}$, this means that we are thinking of the ground category as the category of $E_{\infty} k$-modules rather than the category of $k$-modules.

From the first point of view we see that $M \otimes K$ and $\operatorname{Hom}(K, M)$ are $A$-modules if $M$ is an $A$-module and $K$ is a $k$-module. From the second point of view, we see that $M \otimes L$ and $\operatorname{Hom}^{\bigotimes}(L, M)$ are $A$-modules if $M$ is an $A$-module and $L$ is a $\mathbb{C}$-module.

To develop the cell theory of $A$-modules, we need a free functor from $k$-modules to $A$-modules, and we already have a free functor from $k$-modules to $\mathbb{C}$-modules, 
namely $K \rightarrow \mathbb{C} \otimes K$. The following observation gives a free functor $L \rightarrow A \triangleleft L$ from $\mathbb{C}$-modules to $A$-modules.

Lemma 3.1. For $a \mathbb{C}$-module $L$, define $A \triangleleft L$ to be the pushout displayed in the diagram

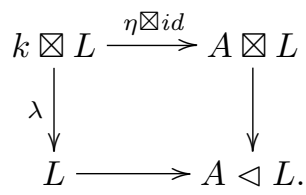

Then, for A-modules $M$,

$$
\mathscr{M}_{A}(A \triangleleft L, M) \cong \mathscr{M}_{\mathbb{C}}(L, M) .
$$

Lemma 3.2. For a $k$-module $K$, define an A-module $F K$ by

$$
F K=A \triangleleft(\mathbb{C} \otimes K) .
$$

Then, for A-modules $M$,

$$
\mathscr{M}_{A}(F K, M) \cong \mathscr{M}_{k}(K, M)
$$

Now recall that we have already constructed the free $A$-module functor for an algebra $A$ over any operad: since the category of $A$-modules is isomorphic to the category of $U(A)$-modules, the free $A$-module generated by a $k$-module $K$ must be $U(A) \otimes K$. We are entitled to the following consequence, which is special to the linear isometries operad. Note that the unit of $U(A)$ determines a natural $k$-map $K \rightarrow U(A) \otimes K$.

Proposition 3.3. For $k$-modules $K$, the natural map $F K \rightarrow U(A) \otimes K$ is an isomorphism of A-modules.

In particular, $F k$ is isomorphic as an $A$-module to $U(A)$. It simplifies matters to assume from now on that $A$ is augmented. This assumption makes it fairly easy to prove the following result, which gives homological control of free $A$-modules, something that is rather difficult to achieve for algebras over general operads.

Proposition 3.4. If $K$ is a cell k-module, then the A-map $\alpha: F K \rightarrow A \otimes K$ induced by the canonical $k$-map $K \rightarrow A \otimes K$ is a quasi-isomorphism. If $K$ is a free $k$-module with zero differential, then $H_{*}(F K)$ is the free $H_{*}(A)$-module generated by $K$.

We can now parrot the development of derived categories via cell modules over DGA's in our more general context of $E_{\infty}$ algebras. For an actual DGA $A$, we have two categories of $A$-modules in sight, namely ordinary ones and $A_{\infty}$ ones. The latter are the same as $U(A)$-modules, and we have the following consistency statement.

Proposition 3.5. If $A$ is a $D G A$, then the quasi-isomorphism $\alpha: U(A) \cong F k \rightarrow A$ is a map of DGA's. Therefore it induces an equivalence of categories from the derived category $\mathscr{D}_{A}$ to the derived category $\mathscr{D}_{U(A)}$. 


\section{The tensor product of $A$-modules}

We have not yet defined tensor products of modules over $A_{\infty}$ algebras. We can mimic classical algebra.

Definition 4.1. Let $A$ be an $A_{\infty}$ algebra and let $M$ be a right and $N$ be a left $A$-module. Define $M \otimes_{A} N$ to be the coequalizer displayed in the following diagram of $\mathbb{C}$-modules:

$$
M \otimes A \otimes N \underset{\mathrm{id} \otimes \nu}{\stackrel{\mu \otimes \mathrm{id}}{\longrightarrow}} M \otimes N \longrightarrow M \otimes_{A} N
$$

where $\mu$ and $\nu$ are the given actions of $A$ on $M$ and $N$.

We construct the derived tensor product $\stackrel{L}{\otimes}_{A}$ by approximating one of the variables by a cell $A$-module.

When $A=k$, our new $M \bigotimes_{k} N$ coincides with $M \otimes N$. We have used the notation $\otimes_{A}$ to avoid confusion with $\otimes_{A}$ in the case of a DGA $A$ regarded as an $A_{\infty}$ algebra.

Proposition 4.2. If $A$ is a DGA and $M$ and $N$ are $A$-modules, then the derived tensor product $M{ }^{L}{ }_{A} N$ is isomorphic in the derived category $\mathscr{D}_{k}$ to the classical derived tensor product $M_{\otimes}^{L}{ }_{A} N$.

The new tensor product enjoys the same formal properties as the classical tensor product over DGA's. We give some examples.

Lemma 4.3. For a right $A$-module $M$ and left $A$-module $N$,

$$
M \otimes_{A} N \cong N \otimes_{A^{\text {op }}} M \text {. }
$$

Lemma 4.4. Let $L$ be an (A,B)-bimodule, $M$ be a $(B, C)$-bimodule, and $N$ be a $(C, D)$-bimodule. Then $L \bigotimes_{B} M$ is an $(A, C)$-bimodule and

$$
\left(L \otimes_{B} M\right) \otimes_{C} N \cong L \otimes_{B}\left(M \otimes_{C} N\right)
$$

as $(A, D)$-bimodules.

Lemma 4.5. The action $\nu: A \otimes N \longrightarrow N$ of a left A-module $N$ factors through a map of $A$-modules $\lambda: A \otimes_{A} N \longrightarrow N$.

The homological behavior of the functor $\otimes_{A}$ is unclear from its definition as a coequalizer, but analysis of its behavior on free modules leads to the following result.

Proposition 4.6. Let $N$ be a cell A-module. Then $\lambda: A \otimes_{A} N \rightarrow N$ is a quasiisomorphism and the functor $M \otimes_{A} N$ of $M$ preserves exact sequences and quasiisomorphisms.

We have a Hom functor $\operatorname{Hom}_{A}^{\bigotimes}$ to go with our new tensor product. It is defined as a suitable equalizer, as in the context of DGA's.

Lemma 4.7. For $\mathbb{C}$-modules $L$ and left $A$-modules $M$ and $N$, there is a natural adjunction isomorphism

$$
\mathscr{M}_{A}(L \otimes M, N) \cong \mathscr{M}_{\mathbb{C}}\left(L, \operatorname{Hom}_{A}^{\otimes}(M, N)\right) .
$$

Analyis of $\operatorname{Hom}_{A}^{\bigotimes}(M, N)$ on free $A$-modules $M$ gives homological control. 
Lemma 4.8. Let $M$ be a cell $A$-module. Then the functor $\operatorname{Hom}_{A}^{\bigotimes}(M, N)$ preserves exact sequences and quasi-isomorphisms in the variable $N$. It also preserves exact sequences of cell $A$-modules in the variable $M$.

We construct the derived functor $R \operatorname{Hom}_{A}^{\otimes}$ by approximating the contravariant variable by a cell $A$-module.

\section{Generalized Eilenberg-Moore spectral Sequences}

Fix an $A_{\infty}$ algebra $A$. Since our derived tensor product and Hom functors generalize those of DGA's, the following definition is reasonable. We are grading by subscripts, with differentials lowering degrees.

Definition 5.1. Define

$$
\operatorname{Tor}_{*}^{A}(M, N)=H_{*}\left(M \unrhd_{A}^{L} N\right) \text { and } \operatorname{Ext}_{A}^{*}(M, N)=H_{-*}\left(R \operatorname{Hom}_{A}^{\otimes}(M, N)\right) .
$$

These functors enjoy the same general properties as in the case of DGA's: exact triangles in either variable induce long exact sequences on passage to Tor or Ext, Tor preserves direct sums in either variable, and Ext converts direct sums in $M$ to direct products and preserves direct products in $N$. The behavior on free modules is

$$
\operatorname{Tor}_{*}^{A}(M, F K) \cong H_{*}(M \otimes K) \text { and } \operatorname{Ext}_{A}^{*}(F K, N) \cong H_{-*}(\operatorname{Hom}(K, N)) .
$$

The crucial point of this generalized definition of Tor and Ext is that we have Eilenberg-Moore spectral sequences for their calculation, just as for DGA's. Following the usual grading convention, write $H_{*}(M)=H^{-*}(M)$.

Theorem 5.2. There are natural spectral sequences of the form

$$
E_{p, q}^{2}=\operatorname{Tor}_{p, q}^{H_{*}(A)}\left(H_{*}(M), H_{*}(N)\right) \Longrightarrow \operatorname{Tor}_{p+q}^{A}(M, N)
$$

and

$$
\begin{gathered}
E_{2}^{p, q}=\operatorname{Ext}_{H^{*}(A)}^{p, q}\left(H^{*}(M), H^{*}(N)\right) \Longrightarrow \operatorname{Ext}_{A}^{p+q}(M, N) . \\
\text { 6. } E_{\infty} \text { ALGEBRAs AND DUALiTy }
\end{gathered}
$$

Let $A$ be an $E_{\infty}$ algebra. The study of $E_{\infty}$ modules works exactly the same way as the study of modules over commutative DGA's.

Theorem 6.1. If $M$ and $N$ are $A$-modules, then $M \otimes_{A} N$ and $\operatorname{Hom}_{A}^{\bigotimes}(M, N)$ have canonical $A$-module structures deduced from the $A$-module structure of $M$ or, equivalently, $N$. The tensor product over $A$ is associative and commutative, and the unit maps $A \otimes_{A} M \rightarrow M$ and $A \rightarrow \operatorname{Hom}_{A}^{\otimes}(A, N)$ are maps of $A$-modules. There is a natural adjunction isomorphism

$$
\mathscr{M}_{A}\left(L \otimes_{A} M, N\right) \cong \mathscr{M}_{A}\left(L, \operatorname{Hom}_{A}^{\otimes}(M, N)\right) .
$$

The derived category $\mathscr{D}_{A}$ is symmetric monoidal under $\stackrel{L}{\otimes}_{A}$, and the adjunction passes to the derived category.

Proposition 6.2. If $M$ and $M^{\prime}$ are cell $A$-modules, then so is $M \otimes_{A} M^{\prime}$.

Again, write $H_{*}(A)=H^{-*}(A)$; it is an associative and (graded) commutative algebra. 
Corollary 6.3. $\operatorname{Tor}_{*}^{A}(M, N)$ and $\operatorname{Ext}_{A}^{*}(M, N)$ are $H_{*}(A)$-modules, and there are natural commutativity and associativity isomorphisms of $H_{*}(A)$-modules

$$
\operatorname{Tor}_{*}^{A}(M, N) \cong \operatorname{Tor}_{*}^{A}(N, M)
$$

and

$$
\operatorname{Tor}_{*}^{A}\left(L \otimes_{A} M, N\right) \cong \operatorname{Tor}_{*}^{A}\left(L, M \otimes_{A} N\right) .
$$

The spectral sequences above are spectral sequences of differential $H_{*}(A)$-modules.

The formal properties we have stated imply many others, just as for classical commutative DGA's and their modules. For example,

$$
\operatorname{Hom}_{A}^{\otimes}\left(M \otimes_{A} L, N\right) \cong \operatorname{Hom}_{A}^{\otimes}\left(M, \operatorname{Hom}_{A}^{\bigotimes}(L, N)\right)
$$

because the two sides represent isomorphic functors on modules. Using this, a formal argument shows that we have a composition pairing

$$
\operatorname{Hom}_{A}^{\otimes}(M, N) \otimes_{A} \operatorname{Hom}_{A}^{\otimes}(L, M) \longrightarrow \operatorname{Hom}_{A}^{\otimes}(L, N) .
$$

This pairing is associative and commutative. It induces a Yoneda product on Ext.

Proposition 6.4. There is a natural, associative, and unital system of pairings

$$
\operatorname{Ext}_{A}^{*}(M, N) \otimes_{H^{*}(A)} \operatorname{Ext}_{A}^{*}(L, M) \longrightarrow \operatorname{Ext}_{A}^{*}(L, N) .
$$

Moreover, there is an induced pairing of Ext spectral sequences that coincides with the algebraic Yoneda pairing on the $E_{2}$-level and converges to this pairing of Ext groups.

Formal duality theory, as developed by Dold-Puppe [2] and others [8] in algebraic topology and by Deligne [1] and others in algebraic geometry, applies verbatim to the present context. We define $M^{\vee}=\operatorname{Hom}_{A}^{\bigotimes}(M, A)$, and we say that a cell $A$ module $M$ is "strongly dualizable" if it has a coevaluation map $\bar{\eta}: A \longrightarrow M \otimes M^{\vee}$ such that the following diagram commutes in $\mathscr{D}_{A}$ :

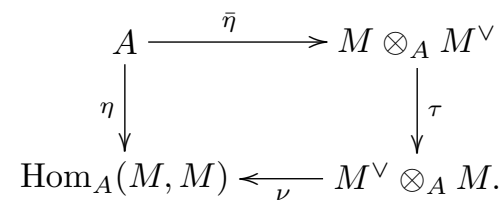

When $M$ is strongly dualizable, various natural maps such as

$$
\rho: M \longrightarrow M^{\vee \vee}
$$

and

$$
\nu: M^{\vee} \otimes_{A} N \longrightarrow \operatorname{Hom}_{A}^{\otimes}(M, N)
$$

induce isomorphisms in $\mathscr{D}_{A}$, exactly as if $A$ were a classical $k$-algebra, without differential, and $M$ were a finitely generated projective $A$-module. The last isomorphism has the following implication, which is an algebraic version of Spanier-Whitehead duality in topology.

Proposition 6.5. For a strongly dualizable $A$-module $M$ and any A-module $N$,

$$
\operatorname{Tor}_{n}^{A}\left(M^{\vee}, N\right) \cong \operatorname{Ext}_{A}^{n}(M, N) .
$$

The following observation, which is due to Greenlees, makes clear that finite cell $A$-modules and their direct summands should be viewed as analogues of finitely generated free and projective modules in ordinary commutative algebra. 
Theorem 6.6. A cell A-module is strongly dualizable if and only if it is a direct summand up to homotopy of a finite cell A-module.

\section{THE LINEAR ISOMETRIES OPERAD}

The algebraic discussion above was all predicated on the good properties of the topological linear isometries $E_{\infty}$ operad. We proceed to define that operad, which was already implicit in Boardman's approach [15] to the stable homotopy category, before operads were invented.

Let $U \cong \mathbb{R}^{\infty}$ be a countably infinite dimensional real inner product space, topologized as the union of its finite dimensional subspaces. Let $U^{j}$ be the direct sum of $j$ copies of $U$. Define $\mathscr{L}(j)$ to be the set of linear isometries $U^{j} \rightarrow U$ with the function space topology. Note that a linear isometry is an injection but not necessarily an isomorphism. The space $\mathscr{L}(0)$ is the point $i, i: 0 \rightarrow U$, and $\mathscr{L}(1)$ contains the identity $1: U \rightarrow U$. The left action of $\Sigma_{j}$ on $U^{j}$ by permutations induces a free right action of $\Sigma_{j}$ on $\mathscr{L}(j)$. The structure maps

$$
\gamma: \mathscr{L}(k) \times \mathscr{L}\left(j_{1}\right) \times \cdots \times \mathscr{L}\left(j_{k}\right) \longrightarrow \mathscr{L}\left(j_{1}+\cdots+j_{k}\right)
$$

are defined by

$$
\gamma\left(g ; f_{1}, \ldots, f_{k}\right)=g \circ\left(f_{1} \oplus \cdots \oplus f_{k}\right) .
$$

The crucial associativity property of $\mathscr{C}$ stems from an associativity property of $\mathscr{L}$ that was first observed by Mike Hopkins. We need a categorical definition in order to state it properly.

Definition 7.1. Working in an arbitrary category, suppose given a diagram

$$
A \stackrel{e}{\underset{f}{\longrightarrow}} B \stackrel{g}{\longrightarrow} C
$$

in which $g e=g f$. The diagram is called a split coequalizer if there are maps

$$
h: C \rightarrow B \text { and } k: B \rightarrow A
$$

such that $g h=\mathrm{id}_{C}, f k=\mathrm{id}_{B}$, and $e k=h g$.

Observe that $\mathscr{L}(1)$ acts from the left on any $\mathscr{L}(i)$, via $\gamma$, hence $\mathscr{L}(1) \times \mathscr{L}(1)$ acts from the left on $\mathscr{L}(i) \times \mathscr{L}(j)$. Note too that $\mathscr{L}(1) \times \mathscr{L}(1)$ acts from the right on $\mathscr{L}(2)$. Let us denote these actions by $\nu$ and $\mu$, respectively.

Lemma 7.2 (Hopkins). For $i \geq 1$ and $j \geq 1$, the diagram

$$
\mathscr{L}(2) \times \mathscr{L}(1) \times \mathscr{L}(1) \times \mathscr{L}(i) \times \mathscr{L}(j) \underset{\mathrm{id} \times \nu}{\stackrel{\mu \times \mathrm{id}}{\longrightarrow}} \mathscr{L}(2) \times \mathscr{L}(i) \times \mathscr{L}(j) \stackrel{\gamma}{\longrightarrow} \mathscr{L}(i+j)
$$

is a split coequalizer of spaces.

Proof. Choose isomorphisms $s: U^{i} \rightarrow U$ and $t: U^{j} \rightarrow U$ and define

$$
h(f)=\left(f \circ(s \oplus t)^{-1}, s, t\right)
$$

and

$$
k\left(f ; g, g^{\prime}\right)=\left(f ; g \circ s^{-1}, g^{\prime} \circ t^{-1} ; s, t\right) .
$$

It is trivial to check the required identities.

Observe that, while covariant functors need not preserve coequalizers in general, they clearly do preserve split coequalizers. This applies to the singular chain complex functor and leads to the following result. 
Proposition 7.3. Let $i \geq 1$ and $j \geq 1$. Then the structural map $\gamma$ of the operad $\mathscr{C}=C_{*}(\mathscr{L})$ induces an isomorphism

$$
\mathscr{C}(2) \otimes_{\mathbb{C} \otimes \mathbb{C}} \mathscr{C}(i) \otimes \mathscr{C}(j) \longrightarrow \mathscr{C}(i+j) .
$$

We use this to construct the promised natural associativity isomorphism

$$
(L \otimes M) \otimes N \cong L \otimes(M \otimes N),
$$

and we claim that both sides are naturally isomorphic to

$$
\mathscr{C}(3) \otimes_{\mathbb{C}^{3}} L \otimes M \otimes N .
$$

Note that $N \cong \mathbb{C} \otimes_{\mathbb{C}} N$. We have the isomorphisms

$$
\begin{aligned}
(L \otimes M) \otimes N \cong & \mathscr{C}(2) \otimes_{\mathbb{C}^{2}}\left(\mathscr{C}(2) \otimes_{\mathbb{C}^{2}} L \otimes M\right) \otimes\left(\mathbb{C} \otimes_{\mathbb{C}} N\right) \\
& \cong\left(\mathscr{C}(2) \otimes_{\mathbb{C}^{2}} \mathscr{C}(2) \otimes \mathscr{C}(1)\right) \otimes_{\mathbb{C}^{3}}(L \otimes M \otimes N) \\
& \cong \mathscr{C}(3) \otimes_{\mathbb{C}^{3}}(L \otimes M \otimes N) .
\end{aligned}
$$

The symmetric argument shows that this is also isomorphic to $L \otimes(M \otimes N)$.

In view of the generality of the previous proposition, the argument iterates to prove that all $j$-fold iterated $\otimes$ products are canonically isomorphic to

$$
\mathscr{C}(j) \otimes_{\mathbb{C}^{j}} M_{1} \otimes \cdots \otimes M_{j} .
$$

When all $M_{i}=\mathbb{C}$, this gives an isomorphism $\mathbb{C}^{\bigotimes_{j}} \cong \mathscr{C}(j)$ of $\left(\mathbb{C}, \mathbb{C}^{j}\right)$-bimodules. We also claimed an isomorphism of left $\mathbb{C}$-modules between $\mathscr{C}(j)$ and $\mathbb{C}$. This arises from the evident fact that if $t: U^{j} \longrightarrow U$ is an isomorphism, then composition with $t$ and $t^{-1}$ give inverse homeomorphisms of left $\mathscr{L}(1)$-spaces between $\mathscr{L}(j)$ and $\mathscr{L}(1)$.

We also used the following observation about $\mathscr{L}$.

Lemma 7.4. The degeneracy map $\sigma_{1}: \mathscr{L}(2) \rightarrow \mathscr{L}(1)$ is an $\mathscr{L}(1)$-equivariant homotopy equivalence.

This does not exhaust the remarkable properties of this truly miraculous operad. The following property is not at all obvious and is not inherited in algebra.

Lemma 7.5. The orbit $\mathscr{L}(2) / \mathscr{L}(1) \times \mathscr{L}(1)$ is a point.

\section{Applications}

The singular cochains of topological spaces can be given $E_{\infty}$ algebra structures. The singular chains of $E_{\infty}$ spaces and thus of infinite loop spaces have evident $E_{\infty}$ algebra structures. Bloch's Chow complexes in algebraic geometry can be transformed into quasi-isomorphic $E_{\infty}$ algebras. The last example leads to one version of a theory of mixed Tate motives $[6,7]$. The first example has been studied by Smirnov [12, 13]. In both cases, much more remains to be done. We turn from algebra to topology to explain fully worked out applications of a parallel theory that we have developed there [5]. 


\section{The STABle homotopy CATEgory}

Since the early 1960's, serious work in stable algebraic topology has taken place in a suitable category of spectra, which plays the role in stable algebraic topology that the category of spaces plays in unstable algebraic topology. Until recently, algebraic topologists always worked, not on the point-set level, but in the stable homotopy category of spectra. Thus associativity, commutativity, and unity were understood as properties that held only "up to homotopy". The reason for this crudity of structure was that we believed that it was impossible to construct a good category of spectra with a smash product that was associative, commutative, and unital on the point-set level, so that it made no sense to ask for precise point-set level algebraic structure.

The topological analog of the algebraic theory that I have described gives such a seemingly impossible category. This theory allows point-set definitions of ring, module, and algebra spectra and thus gives rise to a new subject of stable topological algebra. Most of the important examples of ring spectra in the homotopical sense arise from ring spectra in the new sense: the sphere spectrum, the EilenbergMacLane spectra, the spectra of algebraic and topological $K$-theory, the spectra of cobordism theory, and so on. Many of the examples come from multiplicative infinite loop space theory, which was itself by far the deepest of the earlier applications of topological operads.

For a new style commutative ring spectrum $R$ and $R$-module $M$, it makes sense to construct the localization $M\left[T^{-1}\right]$ of $M$ at a multiplicatively closed subset $T \subset$ $\pi_{*}(R)$ and the quotient $M / I M$ and completion $M_{\hat{I}}$ of $M$ at an ideal $I \subset \pi_{*}(R)$. There are new torsion products and Ext groups that include the Tor and Ext groups of classical algebra and both classical and new homology and cohomology theories in topology as special cases. The new theory has already had many applications $[3,5]$ and is rapidly becoming a standard tool. The applications include:

- Simple new constructions of important spectra that previously were constructed by indirect means and with little algebraic structure.

- Simple constructions of a host of useful spectral sequences - universal coefficient, Künneth, generalized Eilenberg-Moore, etc.

- New and compatible constructions of Quillen's algebraic $K$-theory of rings and Waldhausen's algebraic $K$-theory of spaces.

- A new construction and generalization of topological Hochschild homology and of spectral sequences for its calculation.

- A completion theorem for equivariant complex cobordism and its modules analogous to the Atiyah-Segal completion theorem in equivariant $K$-theory.

\section{A SKETCH OF The Definitions}

Let me give a thumbnail sketch of the modern foundations of stable homotopy theory [4]. Let $U=\mathbb{R}^{\infty}$ be the sum of countably many copies of $\mathbb{R}$, with its standard inner product. A prespectrum $T$ consists of based spaces $T(V)$ for each finite dimensional inner product space $V \subset U$ together with maps $\sigma: T(V) \wedge S^{W-V}$

$T(W)$ when $V \subset W$, where $W-V$ is the orthogonal complement of $V$ in $W$ and $S^{W-V}$ is its one-point compactification. A map $f: T \longrightarrow T^{\prime}$ of prespectra is a collection of maps $f V: T V \longrightarrow T^{\prime} V$ that are strictly compatible with the structure maps. 
A spectrum $E$ is a prespectrum whose adjoint structure maps $\tilde{\sigma}: E(V) \longrightarrow$ $\Omega^{W-V} E(W)$ are homeomorphisms. A map $f: E \longrightarrow E^{\prime}$ of spectra is a map of underlying prespectra. The forgetful functor $\ell: \mathscr{S} \longrightarrow \mathscr{P}$ from the category of spectra to the category of prespectra has a left adjoint $L: \mathscr{P} \longrightarrow \mathscr{S}$. For a based space $X$, define $Q X=\cup \Omega^{q} \Sigma^{q} X$, where the inclusions are given by suspension of maps. The suspension spectrum of $X$ is

$$
\Sigma^{\infty} X=L\left\{\Sigma^{n} X\right\}=\left\{Q \Sigma^{n} X\right\} .
$$

In particular, $S=\Sigma^{\infty} S^{0}$ is the sphere, or zero sphere, spectrum.

The zeroth space $E_{0}$ of a spectrum is denoted $\Omega^{\infty} E$; such spaces are called infinite loop spaces. The functors $\Sigma^{\infty}$ and $\Omega^{\infty}$ are left and right adjoint. More generally, there is a shift desuspension functor $\Sigma_{V}^{\infty}$ that is left adjoint to the $V$ th space functor. We define sphere spectra for integers $n$ by

$$
S^{n}=\Sigma^{\infty} S^{n} \text { if } n \geq 0 \quad \text { and } \quad S^{-n}=\Sigma_{\mathbb{R}^{n}}^{\infty} S^{0} \text { if } n>0 .
$$

We define the smash product of a prespectrum $T$ and a based space $X$ by

$$
(T \wedge X)(V)=T V \wedge X .
$$

We then define $E \wedge X=L(\ell E \wedge X)$. Taking $X=I_{+}$, this gives us a notion of homotopy between maps of spectra. We define homotopy groups of spectra by

$$
\pi_{n}(E)=\left[S^{n}, E\right] .
$$

We say that a map of spectra is a weak equivalence if it induces an isomorphism of homotopy groups. We have a homotopy category $h \mathscr{S}$, in which homotopic maps are identified. The stable homotopy category $\bar{h} \mathscr{S}$ is obtained from $h \mathscr{S}$ by adjoining formal inverses to the weak equivalences.

The essential point is to define the smash product of spectra. On the prespectrum level, we define

$$
\left(T \wedge T^{\prime}\right)\left(V \oplus V^{\prime}\right)=T(V) \wedge T^{\prime}\left(V^{\prime}\right) .
$$

We lift the construction to spectra by use of the left adjoint $L$. The key fact is that $E \wedge E^{\prime}$ is now a spectrum indexed on inner product spaces in $U \oplus U$ rather than in $U$. We call this the external smash product.

Given a linear isometry $f: U \oplus U \longrightarrow U$, we can construct a functor $f_{*}$ from spectra indexed on $U \oplus U$ to spectra indexed on $U$. The composite $f_{*}\left(E \wedge E^{\prime}\right)$ is an internal smash product. Different choices of $f$ give rise to equivalent functors when we pass to the stable homotopy category, and this is what implies the associativity, commutativity, and unity of the internal smash product that we use when defining ring spectra and modules in the homotopical sense.

This smash product is crying out to be reinterpreted in terms of the operad $\mathscr{L}$. There is a "twisted half-smash product" that allows us to glue together all of the $j$-fold internal smash products into a single $j$-fold smash product

$$
\mathscr{L}(j) \ltimes\left(E_{1} \wedge \cdots \wedge E_{j}\right) .
$$

It is equivalent to each of the $j$-fold internal smash products determined by a choice of isometry $U^{j} \longrightarrow U$. This smash product, although canonical, is not associative.

We can define an action of $\mathbb{L}=\mathscr{L}(1)$ on a spectrum $E$ by means of a suitable map $\mathscr{L}(1) \ltimes E \longrightarrow E$. This gives us the notion of an $\mathbb{L}$-spectrum. Examples include all suspension spectra. For $\mathbb{L}$-spectra $E$ and $E^{\prime}$, we can define an operadic smash product

$$
E \wedge \mathscr{L} E^{\prime}=\mathscr{L}(2) \ltimes \mathscr{L}(1) \times \mathscr{L}(1) E \wedge E^{\prime}
$$


that is again an $\mathbb{L}$-spectrum. This smash product is commutative, and the split coequalizer property of the linear isometries operad implies that it is also associative. It is not unital, but there is a natural map $\lambda: S \wedge \mathscr{L} E \longrightarrow E$ of $\mathbb{L}$-spectra that is always a weak equivalence of spectra. It is not usually an isomorphism, but our last surprising property (Lemma 7.5) of the linear isometries operad implies that it is an isomorphism for $E=S$ and for $E=S \wedge \mathscr{L} E^{\prime}$ for any $\mathbb{L}$-spectrum $E^{\prime}$.

We define an $S$-module to be an $\mathbb{L}$-spectrum $M$ such that $\lambda: S \wedge \mathscr{L} M \longrightarrow M$ is an isomorphism. For two $S$-modules $M$ and $M^{\prime}$, we define

$$
M \wedge_{S} M^{\prime}=M \wedge_{\mathscr{L}} M^{\prime} .
$$

The category of $S$-modules is symmetric monoidal under $\wedge_{S}$, with unit $S$, and there is even a function $S$-module functor $F_{S}$ such that

$$
\mathscr{M}_{S}\left(M \wedge_{S} M^{\prime}, M^{\prime \prime}\right) \cong \mathscr{M}_{S}\left(M, F_{S}\left(M, M^{\prime}\right)\right),
$$

where $\mathscr{M}_{S}$ is the category of $S$-modules. We define the derived category $\mathscr{D}_{S}$ by adjoining formal equivalences to the weak equivalences in the homotopy category $h \mathscr{D}_{S}$. This category is equivalent to the stable homotopy category $\bar{h} \mathscr{S}$, and the equivalence preserves smash products and function spectra.

From here, the topological theory is precisely parallel to the algebraic theory. Note the startling fact that, in view of the strict unity property, the topological theory is actually better behaved algebraically than the algebraic theory!

Given the category of $S$-modules, we define an $S$-algebra $R$ by requiring a unit $S \longrightarrow R$ and product $R \wedge_{S} R \longrightarrow R$ such that the evident unit and associativity diagrams commute. We say that $R$ is a commutative $S$-algebra if the evident commutativity diagram also commutes. We define a left $R$-module similarly, by requiring a map $R \wedge_{S} M \longrightarrow M$ such that the evident unit and associativity diagrams commute.

For a right $R$-module $M$ and left $R$-module $N$, we define $M \wedge_{R} N$ by a coequalizer diagram

$$
M \wedge_{S} R \wedge_{S} N \frac{\mu \wedge_{S} \text { id }}{\underset{\operatorname{id} \wedge_{S} \nu}{\longrightarrow}} M \wedge_{S} N \longrightarrow M \wedge_{R} N,
$$

where $\mu$ and $\nu$ are the given actions of $R$ on $M$ and $N$. If $R$ is commutative, then the smash product of $R$-modules is an $R$-module, the category $\mathscr{M}_{R}$ of $R$ modules is symmetric monoidal with unit $R$, and there is a function $R$-module functor $F_{R}(M, N)$ with the usual adjunction. We define $R$-algebras exactly as we defined $S$-algebras, via unit and product maps $R \longrightarrow A$ and $A \wedge_{R} A \longrightarrow A$. All of the standard formal properties of modules, rings, and algebras go over directly to the new subject of stable topological algebra. For example, the smash product $A \wedge_{R} A^{\prime}$ of commutative $R$-algebras $A$ and $A^{\prime}$ is their coproduct in the category of commutative $R$-algebras.

Thinking homotopically, $\mathscr{M}_{R}$ has a derived category $\mathscr{D}_{R}$ that is obtained by inverting the maps of $R$-modules that are weak equivalences of underlying spectra. For an $S$-algebra $R$, the category $\mathscr{D}_{R}$ is not just a tool for the the study of classical algebraic topology, but an interesting new subject of study in its own right. When $R=H k$ for a discrete ring $k, \mathscr{D}_{R}$ is equivalent to $\mathscr{D}_{k}$.

What about examples? There is a notion of an $A_{\infty}$ ring spectrum $E$ that Quinn, Ray, and I defined in 1972 [10]. It is specified by an action of the operad $\mathscr{L}$ on a 
spectrum $E$. Such an action is given by maps

$$
\theta_{j}: \mathscr{L}(j) \ltimes E^{j} \longrightarrow E
$$

such that the evident associativity and unity diagrams commute. If the $\theta_{j}$ are $\Sigma_{j}$ equivariant, then $E$ is said to be an $E_{\infty}$ ring spectrum. It turns out that if $E$ is an $A_{\infty}$ ring spectrum, then $S \wedge \mathscr{L} E$ is an $S$-algebra; if $E$ is an $E_{\infty}$ ring spectrum, then $S \wedge \mathscr{L} E$ is a commutative $S$-algebra. Thus the earlier definitions are essentially equivalent to the new ones, and the earlier work gives a plenitude of examples.

Observe that the operad $\mathscr{L}$ has been exploited in several essentially different ways in this theory.

Descriptively: Thom spectra arise in nature with an action of $\mathscr{L}$ [10]. This observation was the starting point of everything I talked about today.

Constructively: $\mathscr{L}$ acts on Steiner's analog [14] of the infinite little cubes operad; this fact is the starting point of multiplicative infinite loop space theory [11], in which one constructs $E_{\infty}$ ring spectra from $E_{\infty}$ ring spaces.

Foundationally: Properties of $\mathscr{L}$ discovered long after the applications just cited led to our construction of the symmetric monoidal category of $S$-modules.

As this conference has shown, this theory is just one of many in which operads play a central mathematical role. For me, after enjoying their company for 25 years, I still find operads remarkable and delightful creatures, with a knack for springing surprises. Thanks for joining the fun.

\section{REFERENCES}

[1] P. Deligne. Catégories tannakiennes. In The Grothendieck Festschrift, Volume 2, 111-195. Birkhauser, 1990.

[2] A. Dold and D. Puppe. Duality, trace, and transfer. In Proc. International Conference on Geometric Toplogy, 81-102. PWN - Polish Scient. Publishers, 1980.

[3] A. Elmendorf, J. P. C. Greenlees, I. Kriz, and J. P. May. Commutative algebra in stable homotopy theory and a completion theorem. Mathematical Research Letters 1(1994), 225239.

[4] A. D. Elmendorf, I. Kriz, M. A. Mandell, and J. P. May. Modern foundations for stable homotopy theory. In "A handbook of algebraic topology", edited by I. M. James. Elsevier Science. 1995.

[5] A. Elmendorf, I. Kriz, A. M . Mandell, and J. P. May. Rings, modules, and algebras in stable homotopy theory. Amer. Math. Soc. Surveys and Monographs. To appear.

[6] I. Kriz and J. P. May. Derived categories and motives. Mathematical Research Letters, 1(1994), 87-94.

[7] I. Kriz and J. P. May. Operads, Algebras, Modules, and Motives. Astérisque Vol 133. 1995.

[8] L. G. Lewis, Jr., J. P. May, and M. Steinberger (with contributions by J. E. McClure). Equivariant stable homotopy theory.Springer Lecture Notes in Mathematics Vol. 1213. Springer, 1986.

[9] J. P. May. The Geometry of Iterated Loop Spaces. Springer Lecture Notes in Mathematics Vol. 271. Springer, 1972.

[10] J. P. May. $E_{\infty}$ ring spaces and $E_{\infty}$ ring spectra. Springer Lecture Notes in Mathematics Vol. 577. Springer, 1977.

[11] J. P. May. Multiplicative ininite loop space theory. J. Pure and Applied Algebra, 26(1982), $1-69$.

[12] V. A. Smirnov. On the cochain complex of topological spaces. Math. USSR Sbornik 43(1982), 133-144.

[13] V. A. Smirnov. Homotopy theory of coalgebras. Math. USSR Izvestiya 27(1986). 575-592.

[14] R. Steiner. A canonical operad pair. Math. Proc. Camb. Phil. Soc., 86(1979), 443-449.

[15] R. Vogt. Boardman's stable homotopy category. Aarhus University Lecture Notes Series, 21, 1970. 
The University of Chicago, Chicago, IL 60637

E-mail address: may@@math.uchicago.edu 